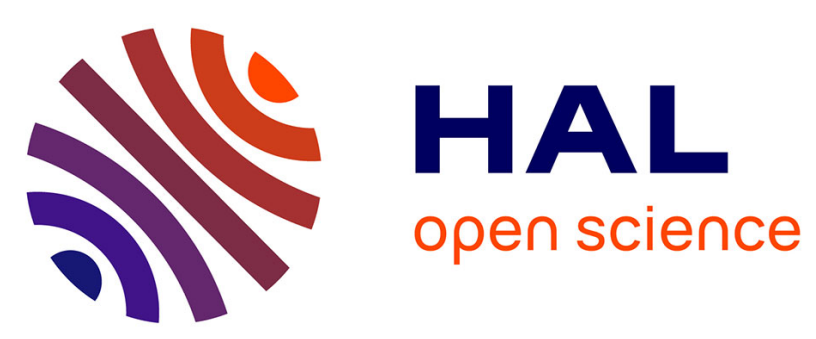

\title{
The Impact of Sensor Noise on Player Experience in Magic Window Augmented Reality Aiming Games
}

\author{
Farjana Z. Eishita, Kevin G. Stanley
}

\section{To cite this version:}

Farjana Z. Eishita, Kevin G. Stanley. The Impact of Sensor Noise on Player Experience in Magic Window Augmented Reality Aiming Games. 14th International Conference on Entertainment Computing (ICEC), Sep 2015, Trondheim, Norway. pp.502-507, 10.1007/978-3-319-24589-8_47 . hal-01758431

\section{HAL Id: hal-01758431 \\ https://hal.inria.fr/hal-01758431}

Submitted on 4 Apr 2018

HAL is a multi-disciplinary open access archive for the deposit and dissemination of scientific research documents, whether they are published or not. The documents may come from teaching and research institutions in France or abroad, or from public or private research centers.
L'archive ouverte pluridisciplinaire HAL, est destinée au dépôt et à la diffusion de documents scientifiques de niveau recherche, publiés ou non, émanant des établissements d'enseignement et de recherche français ou étrangers, des laboratoires publics ou privés. 


\title{
The Impact of Sensor Noise on Player Experience in Magic Window Augmented Reality Aiming Games
}

\author{
Farjana Z. Eishita, Kevin G. Stanley \\ Department of Computer Science, University of Saskatchewan, 110 Science Place, \\ Saskatoon, SK S7N 5C9
}

\begin{abstract}
Augmented reality (AR) requires superimposing digital artifacts on real world scenes. Unfortunately, sensors used to render digital artifacts are subject to noise and imprecision, making the registration difficult in practice. Using a modified version of the Android operating system, we experimentally examined the impact of orientation sensor noise on player experience in three commercial AR aiming games employing different mechanics and input techniques.
\end{abstract}

\section{Introduction}

Game developers have always been early adopters of new technology, pushing the limits of what was technically feasible to craft new experiences for their audiences. With the mobile revolution, electronic play has moved away from the computer console or couch to permeate aspects of everyday life. However, the display area and processing power of handheld devices are limited, meaning players cannot inhabit virtual worlds that are as deep or richly textured as their desktop counterparts. A plausible workaround is to employ the real world as a game board, viewing the world through a smartphone camera, and rendering digital artifacts upon it. We call such games augmented reality (AR) games.

Augmented reality offloads much of the rendering load to the real world. Play environments not longer have to be drawn, they are viewed directly through a digital camera. While the rendering load is much lower, digital artifacts must be correctly placed within the scene, and viewed from the appropriate angle. To accomplish this, sensors initially developed to approximate a phone's position on Earth for navigation and determine phone orientation for screen rotation must now approximate the precision of a virtual camera in a video game. However, unlike the virtual camera used to render scenes in digital worlds, physical sensors are subject to noise, drift and error. In particular, determining the pose of the camera in space so that digital artifacts can be rendered from the appropriate angle, and aiming tasks within the game can be appropriately resolved, is of particular import. In this paper we present an experimental study of the impact of sensor noise on three different aiming games, and show that both noise and input type have an impact on the subjective play experience of participants. 


\section{Related Work}

Location based games employ both orientation for aiming, and fixed real world coordinates. ARQuake [14] is a location-based AR shooting game. The digital artifact rendering occurs based on fiducial vision-based tracking. AR Battle commander [8] is a real-time strategy (RTS) AR game played in an outdoor environment. PasswARG [3] is a geo-tagged treasure hunt game where players find clues provided by the $3 \mathrm{D}$ characters located on POIs (Points of Interest) to find the password to unlock next level. Aiming AR games which soley employ accelerometers or gyroscopes are a significant game research area. Butterfly Effect [6] is an AR game dependent on orientation precision where player wears an HMD and wields a stick rendered as a tornado to collect virtual butterflies. Augmented Galaga [7 is an AR version of the famous arcade game Galaga. ARVe - Augmented Reality applied to Vegetal field [11 is a game for cognitive disable children. LittleProjectedPlanet [4] is an AR prototype of the famous PS3 game 'LittleBigPlanet'. Mind Wrap, Impera Visco, and Penalty kick are games implemented to demonstrate the user interfaces for handheld AR games [12.

\section{Methodology and Experimental Setup}

Many current commercially available MW smartphone games employ aiming with a handset as their primary mechanic. We chose three different games, each employing free aiming - aiming without the aid of visual marker tracking - to ensure that the comparisons reflected differences in aiming modality and not sensing technique. All three games require that players orient the phone to find targets, they differ in how players interact with those targets. All three of these games would be classified as casual arcade games, as they are meant to be played opportunistically and for short durations.

- Chase Wisply [1] is a ghost hunting game using a targeting crosshair.

- Skeeter Beater [9] makes players kill mosquitoes by tapping on the mosquito.

- Droid Shooting [5] requires players shoot robots with a trigger button.

There are several different methods for accessing phone orientation within the Android SDK. These games each use a different software interface. Zero mean Gaussian noise was inserted in a scaled manner in each of these interfaces. Droid Shooting uses accelerometer, Skeeter Beater uses the abstract Orientation sensor which fuses accelerometer, gyroscope and compass readings, and Chase Whisply uses the abstract Rotation Vector, derived from the abstract Orientation sensor.

Zero Mean Gaussian Noise (ZMGN) is a textbook noise model for physical sensors. As multiple small disturbances at various levels of the physical sensor and data acquisition are applied, Gaussian distributions tend to emerge. As the noise is zero mean, it can be added directly to the sensor signal without impacting the average performance. Sensor data error was injected at the operating system level as described below for each of the respective sensors. Through pilot testing 
we configured the standard deviation of sensor error to be at most $2.8 \%$ of the span of the input signal.

We used the Android Galaxy Nexus with AOSP 4.1 in all experiments. We recruited 24 participants (13 male, 9 female; aged from 20-35 years) After each experimental condition, participants completed the PANAS (Positive and Negative Affect Schedule) 2] survey, which measures positive and negative affect, and the IMI (Intrinsic Motivation Inventory) 13] survey, which measures perceived interest, competence, effort and tension.

\section{Results}

Both PANAS and IMI results were analyzed using a multivariate ANOVA. There was a significant difference among the games for players' positive in-game experience $(p=0.033, F=4.001)$ and with noise level $(p=0.001, F=9.637)$. While examining players' negative play experience, both game and noise level effects showed significant difference in variance (game: $p<0.001, F=11.645$; noise: $p<0.001, F=11.242$ ).

Interest and enjoyment of different gameplay was significant between games $(p=0.025, F=4.381)$, and more so with noise $(p<0.001, F=12.407)$. Competence displayed the highest significant difference among all effects (e.g. game, noise level, game-noise level) (game: $p<0.001, F=11.075$; noise: $p<$ 0.001, $F=33.591$, game-noise: $p<0.001, F=9.415)$. Effort only differed by game $(p=0.044, F=3.611)$. Players' tension had significant differences with noise level effect $(p=0.001, F=17.149)$, but not with game.

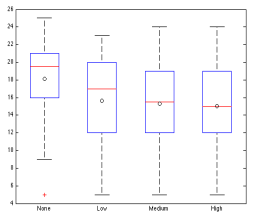

(a) Int. Enj.

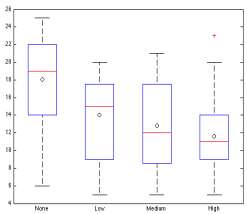

(b) Competence

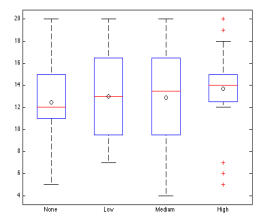

(c) Effort

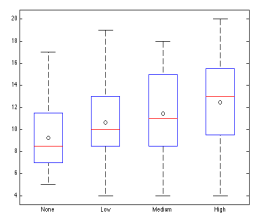

(d) Tension/Pressure

Fig. 1: IMI Responses from Chase Whisply

Although there was no significant difference between Low and Medium level in SK, competence showed significant difference in rest of the levels $(p<0.001$ for relevant pairs). According to the pairwise comparison between subjects, other than Medium and High level of play, the tension/pressure variation was significant $(p=0.006)$. Subjective effort increased with each noise level $(p<0.006$ for relevant pairs). As a result, there was a significant fall of interest and enjoyment of the gameplay with increased noise $(p<0.016$ for relevant pairs $)$ [Figure 2]

For the game Skeeter Beater, almost all the parameters of IMI and PANAS showed significant interaction. In pairwise comparison of between parameters measured by PANAS and IMI, all of them showed significant difference, except 
for the difference between Medium and High noise. Noise impact appeared to saturate after a moderate amount of added noise. Although there was no significant difference between Low level and Medium level plays in SK, competence showed significant difference in rest of the levels ( $p<0.001$ for relevant pairs). According to the pairwise comparison between subjects, other than Medium and High level of play, the tension/pressure variation was significant $(p=0.006)$. Subjective effort increased with each noise level $(p<0.006$ for relevant pairs). As a result, there was a significant fall of interest and enjoyment of the gameplay with increased noise $(p<0.016$ for relevant pairs). Figure 2 provides a box plots of players' experience with SK.

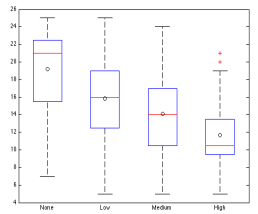

(a) Int. Enj.

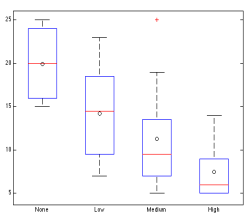

(b) Competence

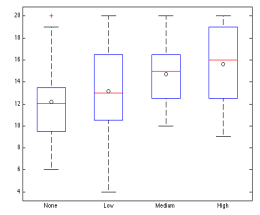

(c) Effort

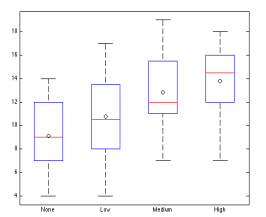

(d) Tension/Pressure

Fig. 2: IMI Responses from Skeeter Beater

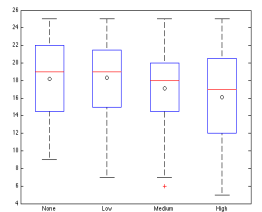

(a) Int. Enj.

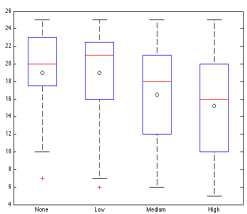

(b) Competence

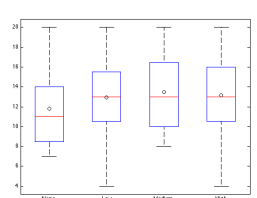

(c) Effort

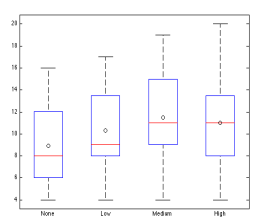

(d) Tension/Pressure

Fig. 3: IMI Responses from Droid Shooting

For the game Chase Whisply, the most significant difference among the different noise levels was observed in competence (pairwise always $p<0.04$ ). Tension and pressure also increased pairwise with noise for No, Low and Medium $(p<0.013)$. For PANAS, only negative feelings were more pronounced with higher noise $(p<0.0106)$.

The least variance was observed in the game Droid Shooting. A noticeable variance was observed in competence $(p<0.0144)$ that went slightly lower with the increment of noise. Overall, a higher tension $\left(p=0.0191, \chi^{2}=9.93\right)$ was observed during DR gameplay with increasing noise [Figure 1 .

\section{$5 \quad$ Discussion and Future Work}

Our primary findings indicate that noise can have a significant impact in MW AR aiming games, that this impact generally increases with increasing noise, and that the magnitude of this impact depends on the type of aiming mechanic 


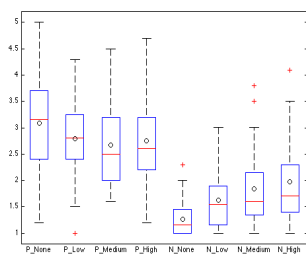

(a) Chase Whisply

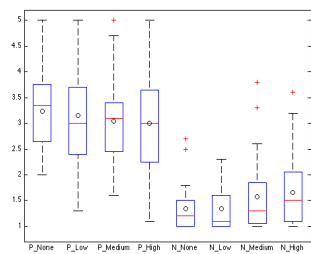

(b) Droid Shooting

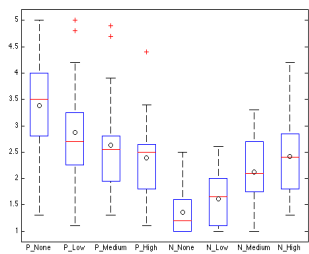

(c) Skeeter Beater

Fig. 4: PANAS Results of all three games

and game mechanics. We suspect a combination of the more difficult aiming technique, which required aiming the phone like a camera while simultaneously tapping the screen, and the negative scoring mechanic caused greater levels of frustration and decreasing competence.

Players suffered more significant decreases in affect and competence in SK than in the other two games. We suspect a combination of the more difficult aiming technique, which required aiming the phone like a camera while simultaneously tapping the screen, and the negative scoring mechanic caused greater levels of frustration and decreasing competence. Because both DR and SK featured moving targets, but DR experienced a much smaller impact with respect to noise, we do not feel that moving targets in these cases were a dominant cause.

The uniformly decreasing competence observed in our experiments is particularly troubling for game designers, as it was noted in [10] that feelings of competency are one of the primary motivators for playing games. The sensitivity observed is particularly important because aiming is fundamental to almost all AR interactions.

While this work has made important contributions to the literature, there are several shortcomings that could be addressed in future work. First, we only focused on aiming games and magic window AR. Second, the games considered, while commercial, were limited in mechanic complexity, narrative scope and artistic design. Finally, while our sample size was large enough given to determine the primary effects, it was skewed towards the university community.

\section{Conclusion}

In this paper we have presented an experimental comparison between the impact of orientation sensor noise on commercial AR games on player experience. We have noted significant impacts of noise in all games tested, but more importantly differential sensitivity to noise.Game developers should consider the interaction mode carefully when designing new interactive experiences to either avoid or masks the noise sensitivity observed, employ more sophisticated filtering techniques and integrate the potential for noise into the overall game design. 


\section{Acknowledgments}

We acknowledge Natural Sciences and Engineering Council of Canada (NSERC), Graphics, Animation and New Media (GRAND) for funding and Ansgar Depping for his cooperation and guidance in the statistical analysis.

\section{References}

[1] Chase whisply, author = Google Play. https://play.google.com/store/apps/ details?id=fr.tvbarthel.games.chasewhisply\&hl=en accessed Feb 17, 2015

[2] Crawford, J.R., Henry, J.D.: The Positive and Negative Affect Schedule (PANAS): Construct validity, measurement properties and normative data in a large nonclinical sample. British Journal of Clinical Psychology 3(43), 245-265 (2004)

[3] Eishita, F.Z., Stanley, K.G., Mandryk, R.: Iterative Design of an Augmented Reality Game and Level-Editing Tool for Use in the Classroom. In: 6th IEEE Consumer Electronics Society Games, Entertainment, Media Conference. p. To appear (2014)

[4] Löchtefeld, M., Str, W., Rohs, M., Krüger, A.: LittleProjectedPlanet : An Augmented Reality Game for Camera Projector Phones. Artificial Intelligence:Proc. of MRIW (2009)

[5] Google Play: Droid shooting. https://market.android.com/details?id=jp.co. questcom.droidshooting, accessed Feb 14, 2012

[6] Norton, M., Macintyre, B.: Butterfly Effect : An Augmented Reality Puzzle Game Marleigh Norton and Blair MacIntyre 2 . Design for Real World Constraints. In: Mixed and Augmented Reality, 2005. Proceedings. Fourth IEEE and ACM International Symposium. pp. 5-6. IEEE Comput. Soc (2005)

[7] Park, A., Jung, K.: Augmented Galaga on Mobile Devices. Flying pp. 888-897 (2007)

[8] Phillips, K., Piekarski, W.: Possession techniques for interaction in real-time strategy augmented reality games. In: Proceedings of the 2005 ACM SIGCHI International Conference on Advances in computer entertainment technology - ACE '05. p. 2. ACM Press, New York, New York, USA (2005),

[9] Google Play: skeeter. https://play.google.com/store/apps/details?id=com. cogtactics.skeeterbeater\&hl=en accessed Dec 17, 2014

[10] Przybylski, A.K., Rigby, C.S., Ryan, R.M.: A motivational model of video game engagement. Review of General Psychology 14(2), 154-166 (2010),

[11] Richard, E., Billaudeau, V., Richard, P., Gaudin, G.: Augmented Reality for Rehabilitation of Cognitive Disabled Children: A Preliminary Study. 2007 Virtual Rehabilitation (2), 102-108 (Sep 2007),

[12] Rohs, M.: Marker-Based Embodied Interaction for Handheld Augmented Reality Games Marker-Based Embodied Interac-. Virtual Reality 4(5) (2007)

[13] Ryan, R.: Control and information in the intrapersonal sphere:An extension of cognitive evaluation theory. Journal of Personality and Social Psychology (43), 450-461 (1982)

[14] Thomas, B., Close, B., Donoghue, J., Squires, J., Bondi, P.D., Piekarski, W.: First Person Indoor/Outdoor Augmented Reality Application: ARQuake. Personal and Ubiquitous Computing 6(1), 75-86 (Feb 2002), 